\title{
Interaction Dynamics in Collaborative Design Discourse
}

\author{
Feniosky Peña-Mora \& Karim Hussein* \\ Intelligent Engineering Systems Laboratory, Department of Civil and Environmental Engineering, \\ Massachusetts Institute of Technology, Cambridge, Massachusetts 02141, USA
}

\begin{abstract}
Computer conferencing is an important emerging technology that promises to have dramatic effects on coordinating the development of large-scale engineering projects. The technology provides instant inexpensive access to all those involved in a project, thereby reducing project costs and enhancing quality. This article outlines key principles that are necessary to realize the full potential of computermediated communication and coordination of group work in the architecturelengineering/construction (AEC) industry. The approaches proposed are based on an analysis of collaborative design discourse data in physical meeting settings. The interactions of group members have been modeled, and the results have been statistically verified. Based on this model, deficiencies in current computer-mediated communication are outlined, and a set of principles for effective conferencing systems is formulated to address these defficiencies. Implementations of these recommendations have been integrated into a distributed collaborative design tool being developed by this research group.
\end{abstract}

\section{INTRODUCTION}

Effective collaboration in large-scale engineering projects requires efficient management of information flow and skillful coordination of group activities. These issues are particularly accute in the architecture/engineering/construction (AEC) industry, where the parties involved in the design and construction process are necessarily multidisciplinary and represent several hundered different specialized design, contracting, and supply firms. Participants in the construction typically work independently while making decisions that inevitably affect the other participants in the construction process. Fur-

* To whom correspondence should be addressed. thermore, firms are generally in different locations and also can be far from the site. Hence coordination and communication are a central component of all major AEC endeavors.

Coordination and design meetings occupy approximately $40 \%$ of an engineer's time. ${ }^{6}$ A large percentage of that time is spent in arranging meetings and commuting to meetings. Reducing meeting time and reducing meeting overhead (organization, scheduling, and commuting time) can greatly reduce costs and enhance product quality (due to increased and more timely communication throughout the life cycle). Video conferencing and other telepresence systems have been experimented with to reduce meeting overhead and enable parties to meet without being physically colocated. Several American and Japanese firms have set up links between site offices and headquarters to provide headquarters with up-to-the-minute information on site conditions and enable redesign if necessary. Currently, the sites have been concerned primarily with acquiring and transmitting information with very limited interaction between the site and the head office. This communication is inefficient in the construction process because the site engineers usually know more information regarding the site that is not directly relayed through structured reports. The current video conferencing systems that have been tested are unable to support the dynamic group interactions necessary in design and coordination processes between the site and main offices. $^{20}$

Supporting distributed group interaction to enhance the product quality and improve competitiveness in the AEC industry requires enhanced communication links throughout the design and construction life cycles. The necessary communication links include structured information (e.g., blueprints) and free-form conversation (e.g., design discussions). Computer communication offers an inexpensive communications bridge among the participants in the development of a constructed facility. Significant research has been 
devoted to the encoding and transmission of structured communication via computer. This includes work in computeraided design (CAD), product and artifact modeling (e.g., STEP), work flow processing (e.g., Lotus Notes), and scheduling. However, limited attention has been devoted to the simplest of communication mechanisms, namely, interaction in the form of discussion and coordination in meetings.

A study of group dynamics in problem-solving meeting environments was performed by our research group to determine the elements of physical discussion and coordination meetings that needed to be replicated in a computer-mediated environment. Data from these experiments were subjected to a detailed analysis of the discourse in order to determine the forms of communication exhibited in a group interaction. The interaction patterns were modeled, and the data were further classified to determine the conversation elements that can be conveyed through the existing audio and video communication systems and the elements that required additional communication channels. The focus was particularly on information flow and on the transfer of speaking role, because they are the two critical variables in the dynamic analysis of discourse. After these elements were determined, techniques for representing them were developed to allow effective interaction in a distributed computer-mediated environment.

The results of the study were used to develop a set of recommendations and a user-interface prototype for coordination in computer-supported collaboration tools for distributed group problem solving. These recommendations are based on the discourse mechanisms meeting participants use to assert their control over the floor in a problem-solving meeting setting, thereby coordinating group work. Floor control is the critical process that affects the dynamics of group collaboration because it restricts the amount of information flow within a group interaction, thereby greatly influencing the design outcome (common pitfalls of group interaction are lack of group focus, caused by distracting floor interjections, and limited exploration of design space due to the monopolization of the floor by particular individuals). Hence a computer-mediated communication system must be able to recognize transitions in floor control and correct errant group dynamics to ensure effective group results and member satisfaction. A core infrastructure for floor control in a distributed setting also has been developed and is presented in the final section. Effective floor control enhances the group problem-solving process and eliminates the need for expensive facilitation services. The following subsection provides a brief description of key aspects of human communication and an elaboration on the concept of floor.

\subsection{Understanding communication}

This subsection presents a brief background on group communication with particular emphasis on the transition of the speaking state (the floor) from one participant to the other. Two key characteristics of efficient meetings (i.e., increased information flow and equal participation of individuals) greatly depend on efficient floor transfer policy. In fact, floor control policies are the principal concern of meeting facilitation strategies. ${ }^{21}$

The concept of floor represents the speaker state within a group discourse. Floor* commonly refers to the right of a member to communicate to a group (e.g., the project manager addressing contractors); alternatively, the term also has been used to refer to the topic of focus in a group discourse (Edelsky, 1993). ${ }^{8}$ For the purposes of this research, floor will refer to the right of a member to communicate to the group.

Several techniques have been proposed to enhance the floor transition process in task-oriented group work, including formal methodologies for facilitation ${ }^{11,41}$ and a fruitful business in group process consulting. ${ }^{36,37}$ The control of floor has been shown to affect power dynamics within a group, and repressive floor control policies can stifle innovation and creativity in a group. ${ }^{31,44}$ Furthermore, ineffective floor policies may lead to frustration, anxiety, and conflict within the group..$^{25}$

In order to enhance the floor transition process, there are two main issues in group dynamics that this article addresses. The first is to investigate the possibility of extending current work on dyadic ${ }^{\dagger}$ turn-taking theories ${ }^{7,14,43}$ and applying them to group floor control in a task-oriented setting, such as a change negotiation meeting in a civil engineering project, using discourse analysis. Most research on turn taking does not take into consideration the situation when more than two persons are conversing. This article argues that the two phenomena are different and use diverse modalities and various discourse elements. In addition, a dyadic model will not work in large-scale engineering projects where a negotiation or design meeting rarely involves only two parties. However, the current work on dyadic turn taking offers a good ground to pursue further analysis for group activities. The second problem addressed by this research is the derivation of a model for floor transition based on observed data and on the discourse analysis influenced by turn taking. The model is based on a consistent description of the various states that a group will experience while exercising floor control. The validity of the derived model will be shown by mapping some of the concepts back to the actual data. This model will then become the basis for a set of requirements for computer conferencing systems to be used on problem-solving distributed meetings in civil engineering projects.

\footnotetext{
* floor (n.) (1) the part of a legislative chamber, auditorium, etc. where the members sit and from which they speak; (2) the right of one member to speak from such a place in preference to other members. Random House College Dictionary, 1995 edition.

$\dagger$ Two-person interactions.
} 


\subsection{Article outline}

The following section provides the relevant background information on group dynamics as well as synchronous distributed communication. Section 3 provides a scenario from the AEC industry to illustrate the concepts discussed in this article. This is followed by Section 4, which describes the methodology used to gather data to use as a basis for deriving the models of floor control, which are described in Section 5 . This is followed by a set of recommendations that any conferencing system that supports the group dynamics present in civil engineering interactions must satisfy. A prototype conferencing system and a floor control infrastructure are also presented in this section. Concluding remarks and future work are presented in the final section.

\section{BACKGROUND ON INTERACTION DYNAMICS}

For the past three decades, linguistics research in discourse has analyzed interpersonal communication and conversation. Most of the literature focused on face-to-face dialogues or dyadic conversations. ${ }^{7,35}$ An overview of the research related to turn taking is presented below, together with comments on relevant issues that have not yet been explored.

Thorisson ${ }^{43}$ provides a comprehensive overview of research on dialog structures and their discourse elements. Thorisson argues that turn taking is crucial for both negotiation and clarification, since it controls the flow of the conversation and hence restricts the amount of information exchanged in an encounter. Thorisson also shows that backchannel is critical in conversations to signal auditor acknowledgment and understanding. According to McNeill and Goodwin, ${ }^{14,23}$ back-channel helps in information exchange to support the interaction and assists in moving along the right path. Usually back-channels are listener utterances that do not interrupt the speaker; when back-channels interrupt the speaker's flow, they may indicate a request for turn.

Furthermore, Thorisson shows that gaze is an important component of a conversation, because not only does it reflect the person's attention or mental activity but also a person might look at an object or other person during a conversation that will provide some deictic* information. ${ }^{3}$ Furthermore, gaze is used to signal the beginning (looking away from the auditor) and end of a turn (looking toward the auditor to pass the turn).

Duncan ${ }^{7}$ offers a good structural analysis of human dialogues but focuses mostly on the nature of the signals between

\footnotetext{
* Pointing or referential gestures, e.g., "place that object here," where "that" is accompanied by a pointing gesture toward a specific object and "here" is accompanied by another pointing gesture that directs the conversing partner to a particular location nearby.
}

the speaker and the auditor. Turn taking in Duncan's opinion (termed the speaker-turn system) includes signals from the speaker, back-channel signals (words such as "uhuh" or nodding the head) from the auditor, and some other state attributes (like the nature of utterances and body motion).

Speaker signals include turn signals, within-turn signals, and continuation signals. The turn signals are signals that the speaker resorts to to request a turn (such as raising a hand or interjecting). The speaker might use cues, which can be intentional, content-based, syntax, paralanguage, and body motion. The latter include gestures that the speaker uses to take turn (e.g., gesturing at a blueprint of the site while others are discussing it, thereby interrupting their field of vision). On the other hand, within-turn signals mark the ending of a turn or utterance and consist of both paralingual or gesture cues (these include dropping off of speech volume or pitch as well as gazing at the other participant). Finally, the continuation signals can occur at the beginning of a speaker turn or at the beginning of a speech unit (these include looking away from the other participant and commencing of gesturing).

Goodwin ${ }^{14}$ showed that gazing is crucial for the speaker during his or her turn of speech. There are several instances when the speaker resorts to gazing to either bring attention to or restart some phrases. It is believed that at the start of each turn, there is a high chance that the auditor will gaze at the turn taker. At some point during the conversation, the person speaking uses several cues to bring the auditor's gaze toward himself or herself. This effect of restarting is used to secure or request the gaze of a hearer. People do this by either lengthening some words, repeating, or creating more pauses. Goodwin showed too that a speaker uses similar techniques to secure the gaze of multiple recipients.

Most of the current research has focused on turn taking as a speaker or auditor state of dialogue; there has been little work on actually defining a good structure or framework on the characterisitcs of a turn (boundaries of when it starts, how it starts, and how one can lose one's turn). Furthermore, there has been limited work on the discourse analysis of group discourse that is crucial for this work to be useful in the AEC context. The work described earlier has been limited to dyadic conversation.

Further work has been conducted in the CSCW (computersupported cooperative work) community in comparative studies of group activity with and without the use of computer mediation. Notably, Olson and coworkers ${ }^{30}$ discuss the changes in group process related to the addition of a shared editor in a computer-augmented meeting room. This work found that the quality of group design improved with the addition of the shared tool; however, the group members were less satisfied with the process. Furthermore, the article asserts that computer-based structuring for problem solving is not necessary and simpler groupware systems are more effective. Most of these early field studies ${ }^{10,16,28,40}$ used synchronous group 
tools that augmented traditional meeting rooms or used very low bandwidth communications. Our research argues that explicit computer coordination of group processes is necessary in a telepresence environment with multimedia support. This is due to the synchronous form of the real-time communication and the overload caused by the high-bandwidth interaction if all participants are equally engaged in the interaction. Furthermore, typical social protocols do not hold true in computer-mediated communication, where there is no physical interaction.

We believe that understanding of group interaction and enhancing the process are critical in developing recommendations for the adoption of computer-mediated communication technologies. This is especially critical in the AEC industry, where there are many parties involved that are multidisciplinary and do not usually work in close proximity.

\section{A SAMPLE TASK SCENARIO}

The following scenario is a fictitious description of a design task that was set by a state's highway department (HD) after a serious accident that damaged a bridge.

On Monday morning, a freighter crashes into the support pier of a bridge crossing a major waterway in a large city. The pier is severely damaged, and the bridge is no longer guaranteed to be structurally sound. This bridge lies on a major artery into the metropolitan area, and a solution needed to be formulated quickly to restore a normal flow of traffic in the city. The owner (HD) convenes a meeting with several experts in the field, including a structural engineer, a traffic engineer, and a contractor to discuss possible solutions. The discussion involves an analysis of the situation and an initial investigation phase where all the parties involved collect data regarding the site, the extent of the damage, and the traffic flow patterns in the area. Several design alternatives are proposed by each of the individuals at the following meeting.

The design proposals are meant to address the four key concerns: public safety and the risk associated with the accident, cost of repair, time and space for repair, and traffic flow reduction or redirection. Given that the bridge can carry a minimal load, several proposals are submitted by the parties to this design negotiation detailing construction alternatives and various traffic redirection patterns.

Throughout the second meeting, the alternatives are discussed, and the initial remedies are rejected due to their high cost, their effect on traffic flow in the area, or their destruction of the aesthetics and symmetry of the bridge. After a heated discussion over three days involving significant compromise among all the parties involved, a preliminary design is ratified. The final design is presented by the structural engineer, and minor adjustments are made in the final meeting. The design is then committed, and the contractor begins the field work.

The scenario just described exemplifies some of the components in critical AEC design meetings. These meetings typically include the following key factors:

1. Urgency: Many design processes in the AEC industry have significant time pressures. Although most design sessions will not have the urgency of the emergency just described, almost any design task has some degree of urgency, and this becomes a critical factor in the design process.

2. Role definition: Each of the members of a design team brings in his or her own interests based on his or her professional experience or personal bias. The efficient combination of these experiences is necessary in the generation of an effective solution.

3. Discovery phase: A stage in which all the meeting participants acquire and analyze all available data regarding the design situation.

4. Brainstorming: In the initial phases of design, several proposals are generated by the participants.

5. Ranking/refinement: Given the initial set of proposals, the engaged engineers will determine collectively the important components of the design options and prioritize the design factors.

6. Detailed design: A final design is generated based on the earlier refinement process and is adjusted by all members of the group.

Having delineated the design process, this research effort is interested in determining mechanisms to support this process and enable conducting such meetings with computermediated communication and reduce design cycle time. Hence an analysis of the standard physical meeting is necessary to determine the modes of communication among the participants. Further analysis of this communication scenario will determine requirements for the computer support of the design process described earlier. The experimental setup and the analysis process are described in the following section.

\section{EXPERIMENTAL METHODOLOGY}

Given the characteristics of AEC design meetings described earlier, an appropriate experimental process was developed. The experimental meeting process needed to elicit the appearance of the following criteria: urgency, a discovery process, varying roles, a ranking and refinement process, and a detailed design process. The experiment chosen was not particular to the AEC domain; however, it was a standard group dynamics simulation that magnifies the key factors described earlier in order to clearly identify the discourse characteristics in this setting.

Data were collected on several group meetings among four to five graduate civil engineering students with an average of 2 years of field experience (using the simulation exercise described below); the data were transcribed and annotated, followed by a discourse analysis of the annotations. A data- 
driven preliminary model (Section 5) was then generated, and the model was verified with the available data.

The data set chosen for analysis was gathered from the following task-oriented group exercise:

A group of students (four to five persons) was given a simulated survival exercise. The exercise involved a crash of a plane in northern Canada, with the group being the sole survivors. The survivors managed to salvage 15 items from the wreckage, and the members of the group were asked to rank the items according to their importance to their survival.

A preliminary analysis of the group process that naturally evolved from this exercise shows a direct correlation with typical engineering design processes. The situation is clearly urgent due to the life-and-death scenario posed. Although the roles of the group members are not predefined in the test scenario, clear roles emerged during the exercise. A group leader emerges, and several advocates for particular solutions also evolve. Although survival is the goal of the exercise, each individual places a different weighting on the four core survival principles in this exercise: food, shelter, communication with rescuers, and reaching the closest settlement. In each of the three groups tested, a member in each of the groups emerged as a champion for one of the survival principles. These champions are similar to the different professionals in the AEC scenario earlier, where each expert represents the interest of his or her field in the problem scenario.

The survival simulation in each of the three cases began with the examination of the map provided and the item list provided. The initial phase of the meeting consisted of each of the members checking each others' facts regarding the situation: their location, the weather conditions, the location of the closest settlement, the terrain, and the use of each of the items on the list. This is synonymous with the discovery phase in the design discussion, where all members of the design team review the extent of the bridge damage and the average daily traffic flow in the area.

Once the discovery process is complete, the group members then attempted to formulate a list. They typically began this process by attempting to brainstorm on the possible uses of all the items on their list (many of the items had multiple uses). They also began to brainstorm on an appropriate course of action given their situation. Similarly in the bridge emergency scenario, the engineers developed several alternatives regarding construction methods and traffic redirection.

In all three groups the members then engaged in ranking the items. They typically chose a coarse ranking and throughout the meeting refined the list until it was agreeable to all those present. (This is a ranking and refinement process as in the alternative negotiation phase in the bridge scenario.) The final list was then drawn up, and a course of action for survival was described. This constituted the final detailed solution design for the survival problem.

\section{MODELING GROUP DISCOURSE}

The detailed discourse analysis of the conversations in the group exercise described earlier revealed two key physical discourse phenomena that govern speaker state transition and information flow in the group conversations. These phenomena are focus of attention and degree of engagement. These two concepts greatly affect the floor transition in group discourse and are described in detail in subsections 5.1 and 5.2.

In addition, two models have been derived from this analysis to describe floor transitions in group discourse. The first model, described in subsection 5.3, describes the state of an individual within the group. For example, this model would characterize the structural engineer's state in a discussion concerning the effect of the accident on the load-bearing limits of the bridge (presumably the structural engineer would either be speaking or engaged in such a discussion). The participant model is complemented with a model that indicates the state of the floor, which is a combined state derived from all participant states (described in subsection 5.4). This model demonstrates the extent of confusion or simultaneous disruptive conversations in the group setting.

\subsection{Focus of attention}

Individuals in a problem-solving group are by nature engaged in a shared task. This task is sometimes embodied by a shared blueprint, document, or whiteboard. Qualitative analysis of the data from the survival exercise shows that group members had attended to a distinct physical space throughout the interaction. This physical space has been termed the focus of attention. Focus of attention is proposed as an additional discourse element that is important in group discourse. This focus is sometimes determined explicitly (as in a parliamentary process where the podium is the main focal point); however, in most group discourse situations the focus emerges from the task discussed and from the particular meeting setting (e.g., a blueprint or physical model placed in the middle of the table in a civil engineering or architectural design meeting).

The focus of attention is a shared space whose manipulation greatly affects the transfer of speakers (floor transition) in a group meeting. Manipulation of the focus may be in the form of deictic and other forms of gestures used within the shared space. In other circumstances, the manipulation consists of writing on, highlighting, or modifying the focus of attention.

The focus of attention varies greatly with the task at hand. When the task is not embodied in a physical space, the focus of attention becomes the gesture space of the member currently controlling the floor (see Yerian ${ }^{45}$ for a description of gesture spaces). In civil engineering meetings, the tasks are typically embodied in a physical space, and identification of a focus of attention is generally simple. 


\subsection{Degree of engagement}

Participants in a meeting exhibit varying degrees of participation or engagement in the active discourse. The status of a participant in dyadic discourse is often classified as a speaker or auditor as in Duncan. ${ }^{7}$ In group discourse, this simple two-state model is often complemented with a middle state referred to as a pending speaker. Observation of freeform group discourse shows that participants cannot simply be classified into these three rigid categories.

Participant state may more accurately be referred to simply as a degree of engagement. With respect to task-oriented group discourse, degree of engagement is defined to be the relative attentiveness or interaction of a participant with the focus of attention. Several factors contribute to an increasing or decreasing degree of engagement with the discourse. These include anxiety of the listener, patience threshold of the listener, interest in the discussed topic, and social and cultural norms of the participant. For example, in the bridge emergency scenario, the structural engineer may be discussing the stress calculations and the maximum load-bearing capacity, in which case the contractor may not be engaged, while the owner and traffic engineer is highly engaged in order to assess the traffic risks of the situation. The continuum of engagement can be further segmented; however, such segmentation does not necessarily provide any additional comprehension of the underlying phenomena. Subsection 5.3 attempts such a segmentation whose states are largely determined by basic discourse elements apparent in each state.

\subsection{Participant model}

The model of each participant engaged in a group discourse is composed of several states ranging from observer to speaker (as opposed to dyadic conversation, where participant roles are classified in a two-state model of listener and speaker). An observer is defined as a member of a group discussion who is not directly engaged in the group discourse. This is generally physically represented by leaning back from the group discourse or by engaging in activities not directly related to the group activity. A speaker in this model is not necessarily engaged in vocal conversation; the speaker is merely the participant in the group discourse who holds the floor (e.g., a person may be demonstrating a traffic flow model without speaking; however, that person has the complete attention of the collaborating members-hence that person has the floor).

The intermediate states between observer and speaker consist of engaged listener, focal interruption, and vocal interruption. These states define varying degrees of engagement (see subsection 5.2) that are attempts to acquire the floor. An engaged listener is characterized by gaze direction, dorsal flexion, and back-channel; he or she is gazing at the focus of attention and is leaning forward in the chair to attract attention. Focal interruption is a subsequent state of engagement in

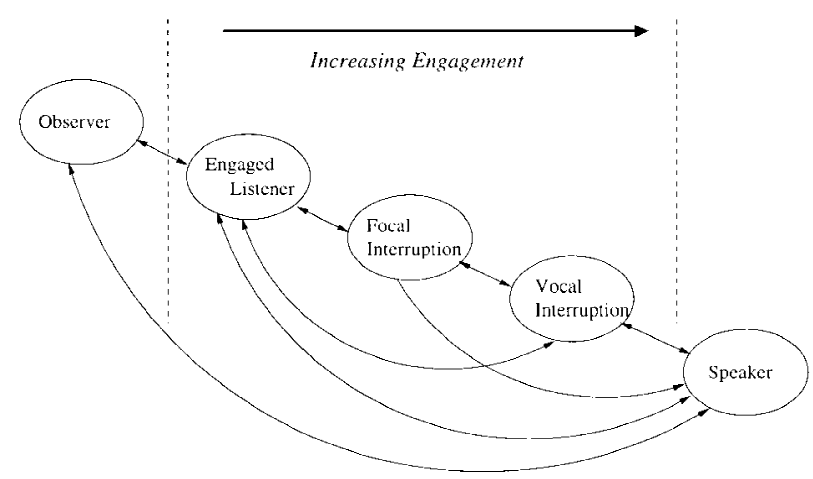

Fig. 1. Participation states.

which the participant interrupts the focus of attention through manipulation of this shared space. Manipulation of this space varies in degrees from simple deictic gestures in the space to physically moving, writing on, or tapping on the shared space. The final intermediate state is vocal interruption. This is the most disruptive form of engagement, which involves the use of verbal techniques to acquire the floor. This involves use of interrupting repair sequences with increasing loudness and verbal interjections using discourse markers such as "oh," "but," "so," and "excuse me."

It is important to note that these states are not clearly delineated and there is clearly a continuum of states from observer to speaker. The categorization just described is a first attempt at clustering degrees of participant engagement. As shown in Figure 1, a particular participant may go through all stages in the model or alternatively may skip over several states; hence it is generally not a serial process. The dominant participant state transitions in the participant model are described in subsection 5.5.

\subsection{Floor model}

The model of floor is simpler than the participant model; however, the floor state transition matrix is significantly more complex. The floor, or active communicating role, is composed of three distinct states: empty, overlapping, and controlled. The empty state is characterized by the lack of intentional* communication between an individual and the group. The overalapping state is characterized by multiple participants in the interrupt or speaker state, thereby signaling a floor transition. Finally, the controlled state is defined to be the state at which there are no floor contentions and only a single participant is in speaker state (Figure 2).

Table 1 shows the various state transitions and the con-

* It is generally difficult to classify intentional communication. However, for the purposes of distributed conferencing applications, all communications are considered intentional. 
Table 1

Floor state transition matrix

\begin{tabular}{rlll}
\hline \multirow{2}{*}{$\begin{array}{r}\text { Initial } \\
\text { state }\end{array}$} & Empty & Overlap & Controlled \\
\cline { 2 - 4 } Empty & $($ null $)$ & $\begin{array}{l}\text { Two or more participants reach } \\
\text { speaker state simultaneously }\end{array}$ & $\begin{array}{l}\text { One participant in speaking } \\
\text { state }\end{array}$ \\
Overlap & Pause, no gesture & $($ null $)$ & $\begin{array}{l}\text { Increased volume, repair se- } \\
\text { quences, spatial control of fo- } \\
\text { cus of attention by one of the } \\
\text { overlapping participants }\end{array}$ \\
Controlled & $\begin{array}{l}\text { Falling pitch, feedback/info } \\
\text { request, pause, no gesture }\end{array}$ & $\begin{array}{l}\text { Back-channel, focal Interrupt, } \\
\text { vocal Interrupt }\end{array}$ & (null) \\
\hline
\end{tabular}

Table 2

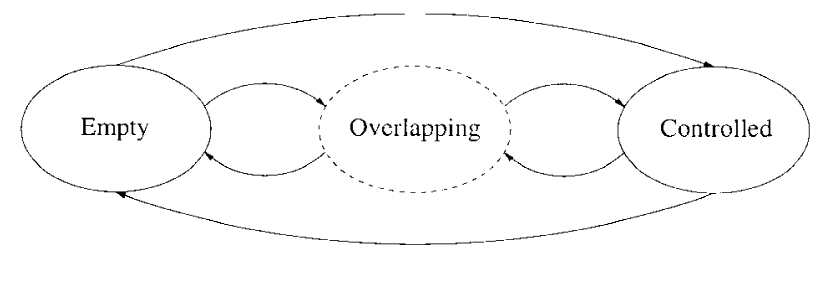

Fig. 2. Floor states.
Trends in floor transition for all three groups

\begin{tabular}{lrr}
\hline Transition & Turns $(\%)$ \\
\hline Observer-engaged & 27 & $7 \%$ \\
Observer-controlled & 38 & $10 \%$ \\
Engaged-controlled & 129 & $34 \%$ \\
Engaged-interrupt-controlled & 186 & $49 \%$ \\
\hline
\end{tabular}

ditions necessary for the transitions that are derived from the participant model and the turn-taking discourse elements discussed in Section 2. The empty floor state is the most difficult to delineate and causes a large portion of the confusion in group meetings. The empty state is characterized by a pause and ceasing of gesturing. However, it is difficult to distinguish between intraturn pause (breathing and thinking) and interturn pause (empty floor). Hence other discourse elements such as the lack of gesturing, falling pitch, and questions posed by the speaker in combination with a speaker pause are better indicators of an empty floor.

\subsection{Model verification}

The participant model hypothesized earlier was verified through an analysis of the experimental data. Transitions among the various states were tabulated for the group and then on an individual basis. The dynamics of the three groups studied were significantly different. The groups chosen for the exercise were culturally diverse, and there were communication difficulties that significantly tainted the data. However, there are significant trends and similarities among all the individuals in the group exercise that allow the generalized model discussed earlier. The floor model was not verified because it is derived primarily from the turn-taking equivalent discussed and verified in Thorisson. ${ }^{43}$

Table 2 shows the general state transitions of the partici- pants in 20-minute segments extracted from each of the three groups. These data were used to test the validity of the degree of engagement hypothesis. The data show that interruptions are used $49 \%$ of the time to signal floor transitions. Furthermore, the engaged participant state was a necessary prerequisite to floor control in $90 \%$ of the floor transitions.

To further classify the forms of interruption into focal and vocal categories, a more detailed analysis was performed on the data shown in Table 2. The second data-gathering effort focused on determining the type and amount of interruptions used by each of the three groups A, B, and C (shown in Table 3 ). The data varied widely among the three groups, which suggests that the dynamics of group interaction are highly dependent on the individuals involved in the group discourse. While the overall use of focal and vocal interruptions was $13 \%$ and $42 \%$, respectively, the use of focal interruptions by individuals in group B was very limited (2\%). The data suggest that vocal interruptions are clearly a valid intermediate state, whereas focal interruptions occur with much less frequency. The data suggest that the degree of focal and vocal interruption is strongly dependent on the structure of the task performed by the group (e.g., engineers examining and modifying a blueprint have a clear focus of attention, while managers discussing corporate strategy may not have a clear focal point-with the possible exception of some graphs indicating market trends).

In this exercise, there was no explicit shared focus of at- 
Table 3

Use of focal and vocal interruptions in floor control in the three experimental groups

\begin{tabular}{|c|c|c|c|c|}
\hline Interjection & $\mathbf{A}(\%)$ & B $(\%)$ & C (\%) & Total $(\%)$ \\
\hline Focal & $2218 \%$ & $2 \quad 2 \%$ & $2115 \%$ & $45 \quad 13 \%$ \\
\hline Vocal & $4839 \%$ & $5053 \%$ & $5037 \%$ & $14842 \%$ \\
\hline Neither & $5243 \%$ & $4345 \%$ & $6648 \%$ & $16145 \%$ \\
\hline
\end{tabular}

tention, except for group $\mathrm{C}$, which had the benefit of a blackboard. The seating arrangement of groups A and B also differed greatly. Group A was seated in a much tighter arrangement, whereas the individuals in group B were more spread out; this may partially explain the limited use of a focus of attention by group $\mathrm{B}$.

Finally, the data were sliced once more to determine the individual influence on group dynamics (it is important to note that the groups were composed of individuals from varying cultural and professional backgrounds). The interjection types were tabulated for each of the individuals in group A (JR, SR, HC and YC) and are tabulated in Table 4. Two individuals dominated the floor in this group, JK and SR; however their use of focal and vocal interruptions varied. While JK's interruptions were spread evenly among focal and vocal, SR hardly used focal interruption. HC exhibited similar behavior to JK, although they are from completely different backgrounds (native Chinese and American). YC interacted minimally with the group. Both YC's and HC's limited interaction may be attributed to their inability to converse comfortably in English. The data are inconclusive regarding the reasons for the use of different mechanisms for interruption. Further controlled studies are required to determine inter- and intracultural use of interruption. This is particularly important given the increasing globalization of business, especially in the AEC industry. However, the data confirm the validity of the focal and vocal interruption states in the participant model because they were present in over $50 \%$ of the floor transitions.

\subsection{Modeling results}

An analysis of interaction patterns in group design discourse was undertaken through an experimental setting described in section 4 that directly mirrors the scenario described in section 3 . The preliminary hypothesis derived was verified through data accumulated from the experimental exercise. This data revealed the important dynamic characteristics of physical meetings. Discourse analysis was used to model the conversation flow (specifically the control of the floor) and the various signals that contributed to a change in the state of the floor. This allowed us to identify the weaknesses of current conferencing systems in supporting group problemsolving interactions such as design meetings and to generate a set of requirements for future systems that are delineated in Section 7. The key results of this exercise are outlined below:

- Participants exhibit multiple levels of engagement in the meeting setting. These engagement levels are critical to the floor transition process because they provide cues to all participants regarding the current state of the floor and the possibility for taking it.

- Interaction among designers is commonly governed by their physical proximity to each other and to the shared element in the meeting room (i.e., the focus of attention, which can be a design specification, a site map, or a simple blackboard). The participants' gaze and manipulation for the physical space surrounding them contribute significantly to the efficient transfer of floor because they make the participants aware of an individual's intent to speak.

- Inefficient interaction patterns (e.g., long divergent conversation or multiple simultaneous discussions) are identifyable through syntactic cues (e.g., number of engaged individuals, number of individuals that are speaking, and increasing delays for individuals attempting to take the floor) in the interaction because floor state can be determined without semantic knowledge of the interaction.

\section{RECENT WORK IN COMPUTER-MEDIATED COMMUNICATION}

There has been a significant amount of research in the area of computer-mediated communication. The work spans multiple disciplines, and hence there are three diverse focus areas in this research field*: electronic meeting systems (EMSs), video conferencing, and shared social spaces. Each of these groups represents a different approach to computer-mediated communication. EMS research focuses on the meeting process and decision support tools for the meeting process. Video conferencing research is concerned with transmitting multimedia data between participants (especially audio and video data). The shared social spaces perspective is concerned with enabling interaction and experience across distance and pro-

\footnotetext{
* This classification is based on the commonalities within the different subgroups. Researchers in all three different fields tend to use multiple vocabularies that can confuse the functionality of the systems.
} 
Table 4

Use of focal and vocal interruptions in floor control segmented by members of group A

\begin{tabular}{|c|c|c|c|c|c|}
\hline Interjection & JK $(\%)$ & SR (\%) & HC $(\%)$ & YC $(\%)$ & Total $(\%)$ \\
\hline Focal & $1022 \%$ & $48 \%$ & $828.5 \%$ & $0 \quad 0 \%$ & $2218 \%$ \\
\hline Vocal & $1533 \%$ & $2154 \%$ & $1243 \%$ & $0 \quad 0 \%$ & $48 \quad 39 \%$ \\
\hline Neither & $2045 \%$ & $1638 \%$ & $828.5 \%$ & $8 \quad 100 \%$ & $5243 \%$ \\
\hline Total & $4537 \%$ & $4134 \%$ & $2823 \%$ & $86.5 \%$ & $122100 \%$ \\
\hline Time(min) & $6.331 \%$ & $9.648 \%$ & $3.317 \%$ & $.84 \%$ & $20 \quad 100 \%$ \\
\hline
\end{tabular}

viding awareness and persistance within a virtual world. The following paragraphs will discuss the salient features of representative systems in each of these areas.

Electronic meeting systems encompass a large body of research dedicated to the support of participants in traditional meeting settings. These systems arose from defense needs for efficient command and control centers. The GroupSystems EMS $^{28}$ and the Xerox Parc Collab project ${ }^{40}$ are among the first such systems developed. Both systems have tools that structure brainstorming and problem-solving processes and enforce interaction controls on the participants within the shared media. However, the control of floor in discussion is governed by regular meeting norms, since all participants are colocated. Olson and coworkers ${ }^{30}$ found that some of these additional process structuring constraints on the collaboration are not necessary and may decrease satisfaction within the work group. Further analysis of the use of these systems and their effect on group work are well documented. ${ }^{10,16,29}$

Initial research on video conferencing focused on the technical aspects of transmitting video and audio data among individuals. Much of the initial work was constrained to twoperson interactions, and a large portion of the work used a telephony paradigm for the interaction. Further developments have occurred rapidly in this field, and most modern systems such as Microsoft NetMeeting, ${ }^{24}$ Intel Proshare, ${ }^{18}$ PictureTel, ${ }^{33}$ and SGI Inperson ${ }^{38}$ provide multiperson interaction and have extended audio and video services to include shared whiteboards, editors, and browsers. However, these conferencing systems lack any appropriate concurrency control mechanisms and are cumbersome to use for group work.

The final area of research in telepresence is devoted to the study of virtual communities and interaction in a virtual environment. Several tools have been developed to provide awareness, persistence, and interaction in cyberspace. The two leading research efforts in this field are TeamRooms ${ }^{34}$ and Worlds. ${ }^{13}$ The primary concern of these research efforts is in the representation of physical concepts of space and place in the virtual world. The environments developed provide richer interaction contexts but are currently constrained by network bandwidth and display technology.

The work described in this article builds on earlier work in all these fields, especially earlier work on electronic support

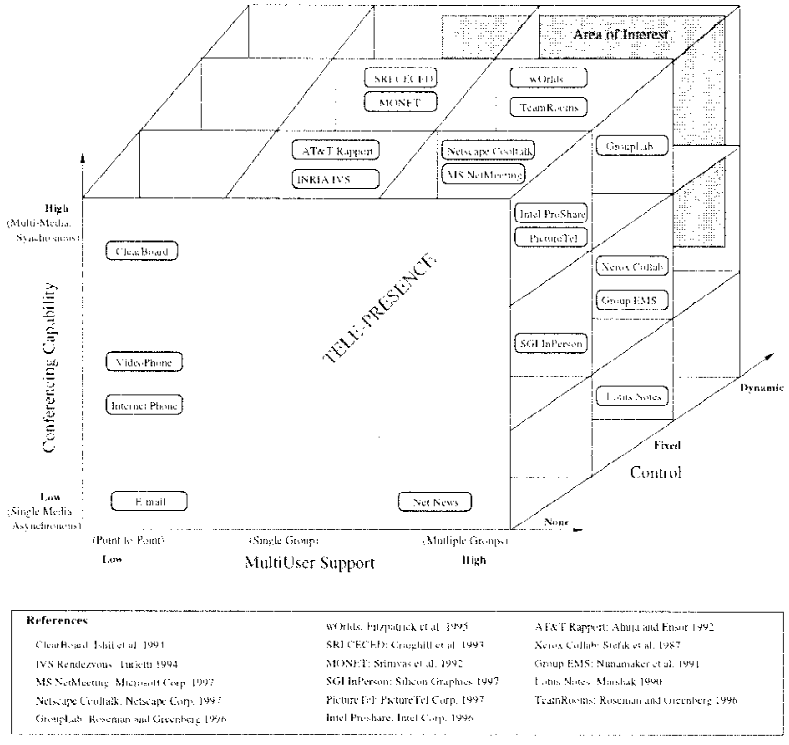

Fig. 3. Overview of computer-assisted communication.

for physical meetings. However, our aim is to extend support mechanisms to distributed meetings using commercial video conferencing technology. This necessitates the introduction of flexible control and interaction support tools to complement the decision support and conferencing infrastructures. The group interaction research described herein, in addition to the recommendations presented, is an initial step in providing such group support.

Some of the current computer-mediated communication systems (both academic and commercial) are classified in Figure 3. The figure delineates the multimedia capabilities of the systems on the $y$ axis. The $x$ axis describes the extent to which these systems support multiple participants in an interaction. Finally, the $z$ axis expresses the degree to which the systems allow effective control of the floor (concurrency control) in collaborative interaction. The core focus of this article is on the $z$ axis, although the existence of multimedia and multiuser support is a necessary prerequisite to the work described herein. 


\section{GROUP DYNAMICS-AWARE CONFERENCING}

The data accumulated in the experiment discussed above as well as previous work in group dynamics ${ }^{2,4,30}$ provide a greater understanding of interactions in group discussion. Several elements of physical interaction are not directly replicable with simple audio and video communication. The elements of engagement and attention discussed in the preceding section are critical in directing the flow of the conversation to facilitate the problem-solving discussion. Given these defficiencies of current conferencing systems, a set of requirements and a core infrastructure for group dynamics-aware conferencing tools have been developed. This section provides an overview of the state of the art in computer conferencing and discusses the mechanisms required to support an effective group discussion in an engineering problem-solving setting.

The analysis of the group interaction data discussed earlier can be performed on two levels. This analysis provides an understanding of the group process and the mechanisms necessary to support it. The lowest level examines the interaction dynamics among individuals in the group. The second, more abstract level is a descriptive underlying floor control strategy (e.g., chairman controlled, democratic/free-form, or lecture) that is either formally acknowledged or dictated by the setting and norms of the interaction.

The models derived in Section 5 suggest several important implications for interaction dynamics in distributed synchronous communication. The models and experimental data outline the key aspects of group discourse that require a physical copresence of the group members. Aspects of a participant's degree of engagement cannot be realized by current conferencing technology ${ }^{9}$ (assuming simple video and audio connections, not including some of the virtual reality systems under development, ${ }^{26}$ which are attempting to simulate the full physical embodiment of individuals in an interactionunfortunately this technology is expensive, cumbersome, and far from being applicable commercially). Motions and movements in engagement are subtle and assume a focus of attention that can be manipulated. Current conferencing systems have a limited notion of shared physical space that may be manipulated as in physical meetings. The experiments conducted have shown that this shared space, along with the visual and physical indicators of participant engagement, is necessary for effective floor transition in multiperson problem-solving meetings.

The following subsection describes the core conferencing architecture and is followed by subsection 7.2, which details infrastructure for floor control developed by this research group. Finally, subsection 7.3 provides a detailed discussion of the user-interface features necessary to satisfy the requirements outlined by the analysis performed in Section 5 in computer-mediated communication tools. A sample implementation is also presented in this subsection.

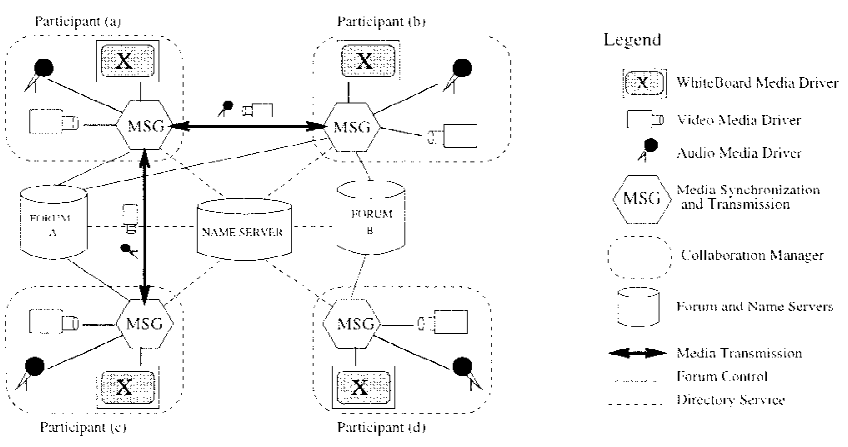

Fig. 4. Prototype conference system architecture.

\subsection{Conferencing system architecture}

A prototype system for group dynamics-aware conferencing has been developed by this research group based on the conferencing guidelines outlined in Section 6. The system provides a distributed communication infrastructure for multimedia interaction. The architecture is shown in Figure 4. The system is composed of a name server that maintains client addressing information, a set of forum servers, and a set of clients. The system provides separate data (shown as a solid line) and control paths (shown as dotted lines). This model allows for controlled interaction among the participants without a communication server bottleneck. Control of communication is achieved by the distribution of data channel keys by the forum server to the clients. Forum servers maintain the meeting process by providing control of meeting interaction and maintaining persistent meeting objects (a more detailed description of the control process is presented in subsection 7.2). The clients are the participant's interface to the communication system. Each client includes a user interface, multiple media device drivers (e.g., whiteboard, scheduling, text, audio, and video), and a message handling system. Various elements of the interface are described in detail in the following subsections. The message handling system is responsible for communication control and synchronization of the multimedia information (see Figure 5). Synchronization is necessary due to the stochastic latency in packet delivery across the Internet. The message system queues incoming data and assembles multiple-media messages into frames that are presented as a single time instant. The synchronization algorithm is presented in further detail in Peña-Mora and coworkers. ${ }^{32}$

\subsection{Control infrastructure for group conferencing}

A communication control model has been developed to support both individual interaction and process control in group interactions. This model is centered around a forum server that acts as the communication control mechanism from the 


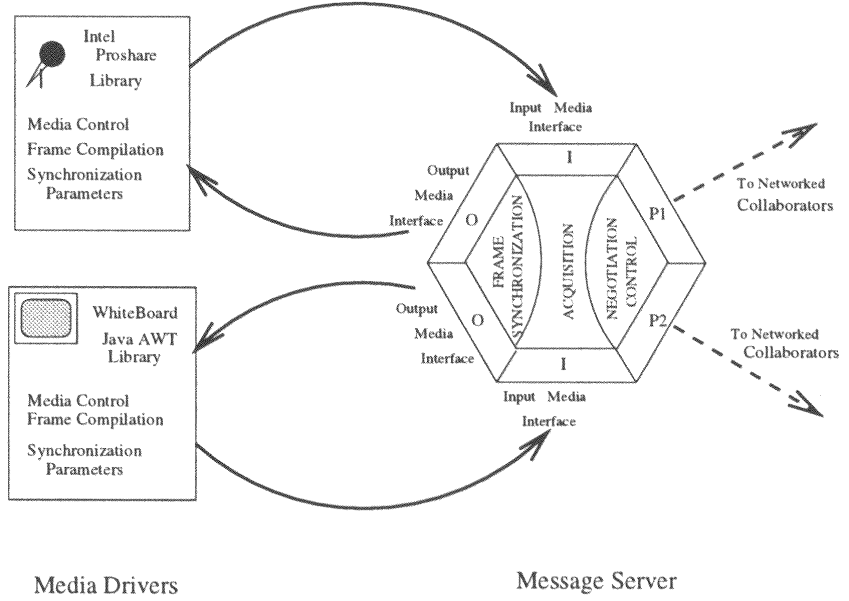

Fig. 5. Message server overview: media drivers for audio and whiteboard devices.

conferencing system. The forum server's primary function is the allocation of communication channels among individuals in the group. Communication among individuals is not channeled through this server but is rather controlled by the forum process.

Forum processes are initiated by an agenda-building tool that allows the definition of meeting membership, meeting control strategies, meeting agenda, and meeting notification. The tool is shown in Figure 6. The meeting may be defined as open (i.e., any person can enter the meeting room) or closed (in which all participants in the meeting must be predefined in the agenda tool). Each meeting member is also assigned particular access rights that include agenda editing, chairperson control, and control of the meeting proceedings. The agenda tool is also used to define the meeting agenda items, which are each assigned a floor control strategy by the meeting initiator. Once the agenda is complete, the system automatically sends notification messages to the participants, and a forum server process is created with the appropriate membership and agenda.

The forum server model is shown in Figure 7. The forum class processes messages from the client systems, which represent each participant in the meeting. The forum class is also responsible for maintaining meeting membership and temporal control of the meeting. This includes meeting notification, agenda traversal, and maintaining and traversing meeting logs. Communication requests received by the forum class from the clients are handled by one of the subclasses of the control class. The control classes contain functions to manipulate tokens* and manipulate the speaker queue. The queue is composed of all members that are interested in ac-

* Tokens are software keys that allow communication between two clients.

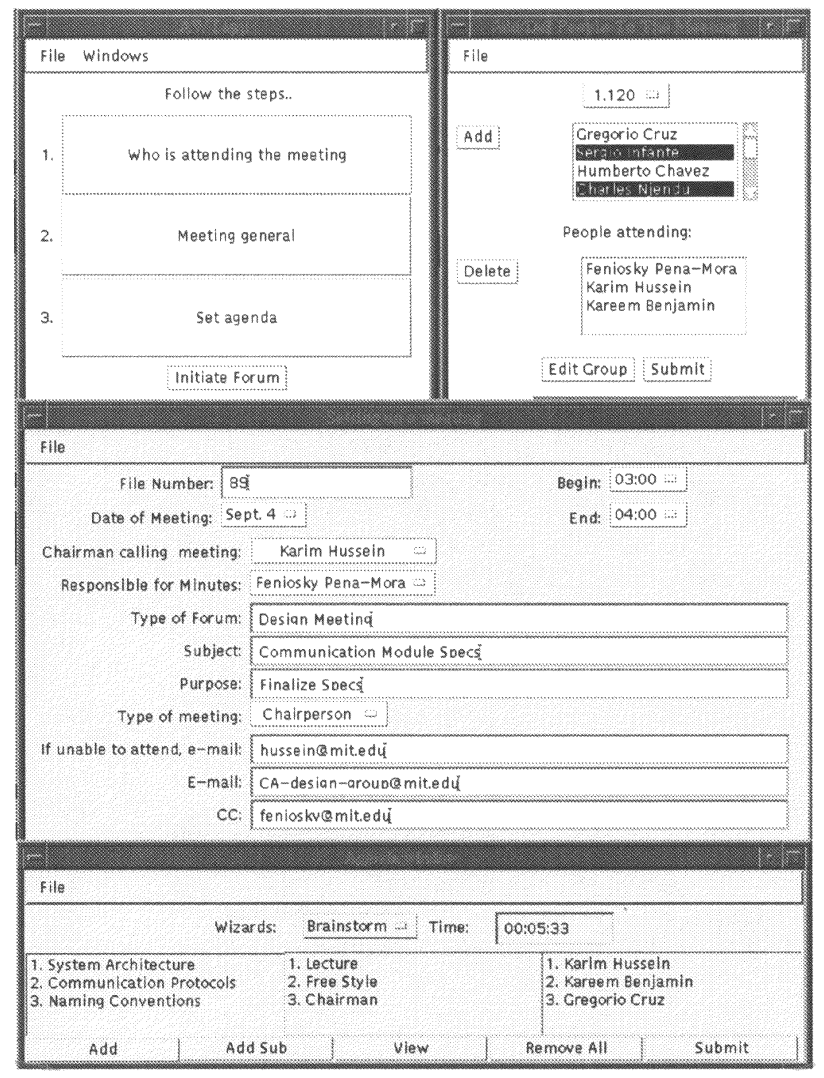

Fig. 6. Agenda-building tool interface.

quiring the floor. The ordering of the queue is based on the particular control strategy used. For example, the chairperson strategy would allow explicit ordering of the queue by the chairperson; the brainstorming queue would simply be a first-in, first-out (FIFO) queue. Ordering of the queue also can be based on more complex inputs provided by the userinterface mechanisms described in the following subsection. This dynamically ordered queue is described in further detail in sub-subsection 7.3.4.

\subsection{User interface for distributed interaction}

The following sub-subsections outline recommendations for user interface design for synchronous distributed communication to support the collaboration process. Sample implementations in the conferencing system developed by this research group are also presented as a mechanism to satisfy these requirements. This conference system includes several extensions that enable voice, visual, textual, and graphic interaction, as well as Web-based shared document browsing. Finally, a scheduling interface to Primavera is included for large-scale collaborations (Figure 8 shows the various tools within the conferencing system). 


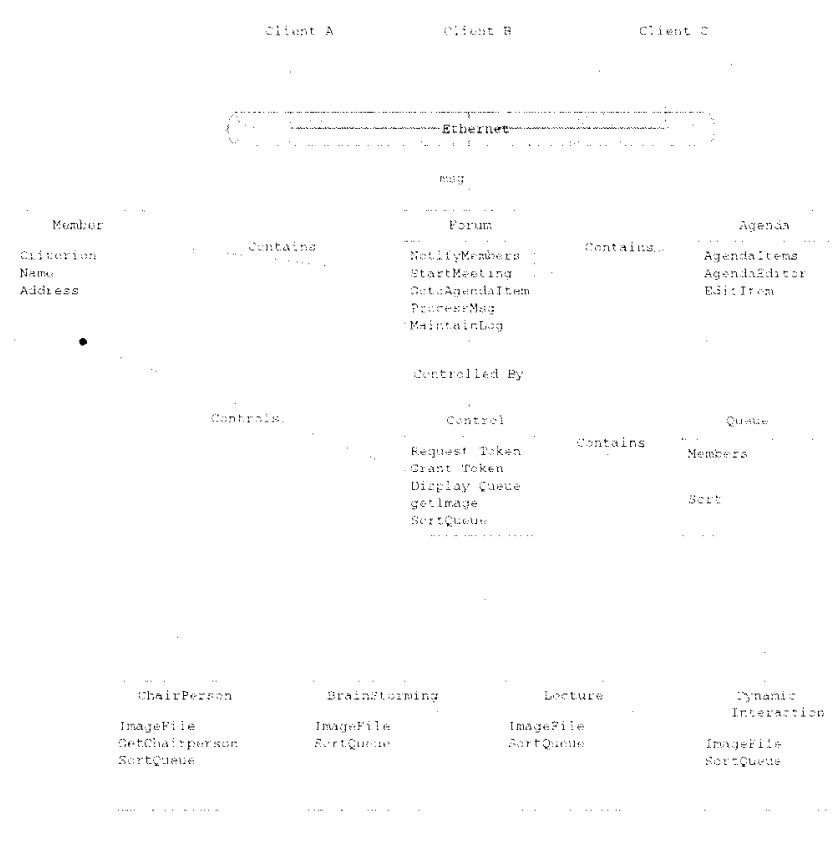

Fig. 7. Control infrastructure model.

Sub-subsection 7.3.1 describes the general sense of space and the complementary concept of place. Sub-subsection 7.3.2 outlines the support of spatial interaction among collaborators. This is followed by a mechanism to support varying degrees of engagement in distributed conferencing. Finally, sub-subsection 7.3.4 develops a macro view of floor transition and control to enable effective facilitation of distributed meetings.

7.3.1 A sense of place. The literature on computer supported work has been engaged in a fruitful discussion regarding the representation of awareness in synchronous group interaction. The notion of place where members meet and share persistent objects has become a growing influence in the CSCW (computer-supported cooperative work) community. ${ }^{12,34}$ It is critical to include mechanisms in the user interface that clearly portray entrance and egress of individuals as well as their relative stance to the others in the meeting. This provides a frame of reference for the collaborators that is essential for effective communication. The implementation chosen by this research group (shown in Figure 8 is one sample mechanism for promoting awareness; other efforts such as Xerox's Placeware ${ }^{15}$ have more elaborate schemes for representing place that are necessary for casual interaction but are less important in formal meetings).

7.3.2 Spatial interaction. A group-aware conferencing tool must support deictic referencing in both gaze and pointing. Hence the tool must have a pointing feature as well as a feature that clearly distinguishes between hearers of conversation and those to whom the conversation is addressed (as dictated by gaze in traditional meeting settings).

Conferencing systems typically provide a large set of interaction tools including whiteboards, text tools, audio, video, and CAD or document sharing. These tools, although useful, can be distracting to the user because they do not provide a clear focus of attention. This does not suggest that the tools should be reduced; instead, it is necessary to include a mechanism for identifying the focal tool of the discourse. This tool becomes the center of floor transition engagement. This research approach is to bring the focal tool to the foreground of the screen or highlight the focal window to represent the focus of attention (see Figure 9).

7.3.3 Degrees of engagement. Since most conferencing tools adhere to a telephony paradigm, a person wishing to speak can only be in two states (dialing or engaged). The results of the research presented in subsection 5.2 clearly indicate that the participant should have greater flexibility in defining his or her intent to take control of the floor. An initial interface that provides this functionality is shown in Figure 9. Furthermore, the pending speaker queue should be prioritized to allow for urgent commentary in an on-line meeting, and the queue should allow simple disengagement from the conversation. Finally, the state of each participant should be visible to all those engaged in the on-line meeting. A threshold is then set for the value of engagement such that the floor changes from controlled to overlapping. This is necessary even during strictly chairperson control conferencing schemes.

7.3.4 Floor control strategy. A final requirement for groupaware conferencing is the notion of floor control strategies (e.g., chairman controlled, brainstorming, and lecture). In regular meetings, a strategy is adopted either explicitly or implicitly due to group norms or due to particular meeting room arrangements. These strategies govern floor control on the macro level; they define a style for a group meeting. Effective choice of floor control strategy can improve the fluidity of the meeting process and enhance the collaborative effort. A toolkit of strategies has been developed by this research effort ${ }^{17,32}$ and has created a knowledge base that maps these strategies to various meeting situations. These strategies are specific to meetings or to individual agenda items. The user-interface representation of three strategies is shown in Figure 10.

A more complex floor control strategy also has been developed based on the interaction inputs provided by the interfaces described earlier. The degree of attention was used to sort the queue of pending individuals. The sort function depended on both the level of engagement and the time that the individual was on the pending queue. The engagement level is given a value from 0 to 1 , and the time on the queue is measured in seconds. An objective function for sorting the 


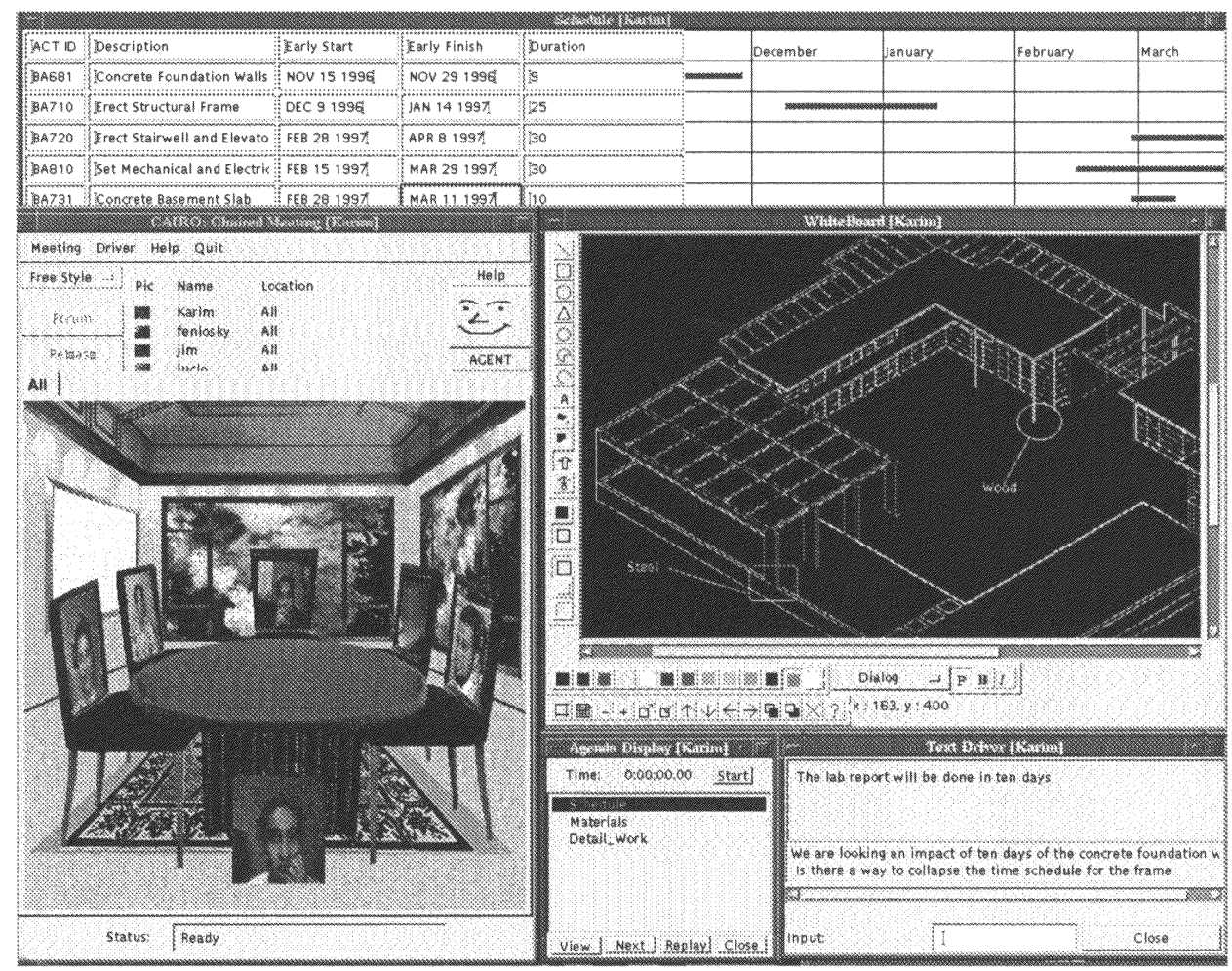

Fig. 8. A complete view of the elements of the CAIRO research effort.

queue is derived by multiplying the two values. This provides a more natural floor transition process and generally is preferred by test users for brainstorming sessions.

\section{CONCLUSIONS AND FUTURE WORK}

Enhanced distributed collaboration promises to be a great influence on the design process of large engineering projects by providing instant access to project information and project engineers. Such distributed environments would significantly improve coordination in the AEC industry and would significantly reduce costs and turn-around time. Effective distributed design environments clearly will be critical components of successful design efforts in the near future.

The study and models presented in this article highlight the necessary features of distributed design environments. Furthermore, the article provides an explanation of the major shortcomings of current computer-mediated multimedia communication systems and presents a prototype system that satisfies dynamic interaction requirements. Through the analysis of physical group design discussion, important factors that allow meetings to run efficiently in physical settings have been identified. These factors were analyzed, and a set of requirements for enhancing coordination and enabling floor transition in distributed computer-supported AEC systems has been presented. This article has stipulated the addition of mechanisms in conferencing systems to represent a sense of place, spatial interaction, degrees of participant engagement, and floor control strategies. The communication system presented is simpler to use and more effective in multiuser distributed collaborative settings because it mimics the natural process of group discourse in physical meeting settings.

Further research remains to be conducted in group dynamics applications in computer-mediated communication. Understanding cultural differences in floor transition is necessary to provide global distributed communications. The data also have shown that different individuals express their interest in acquiring the floor in varying ways. Hence the model proposed in this article may need extensions for multicultural collaboration. Furthermore, the states outlined in the participant model are not the sole manner in which the increasing engagement of a participant may be modeled. The level of segmentation of this continuum of engagement is still an open issue. Finally, the model does not account for parallel conversations that occur often within a group discourse. However, "effective" groups are not supposed to exhibit this type of behavior.

Furthermore, a protocol needs to be devised to reduce participants over- or understating their degree of engagement, thereby reducing individual irregularities. This is not a se- 


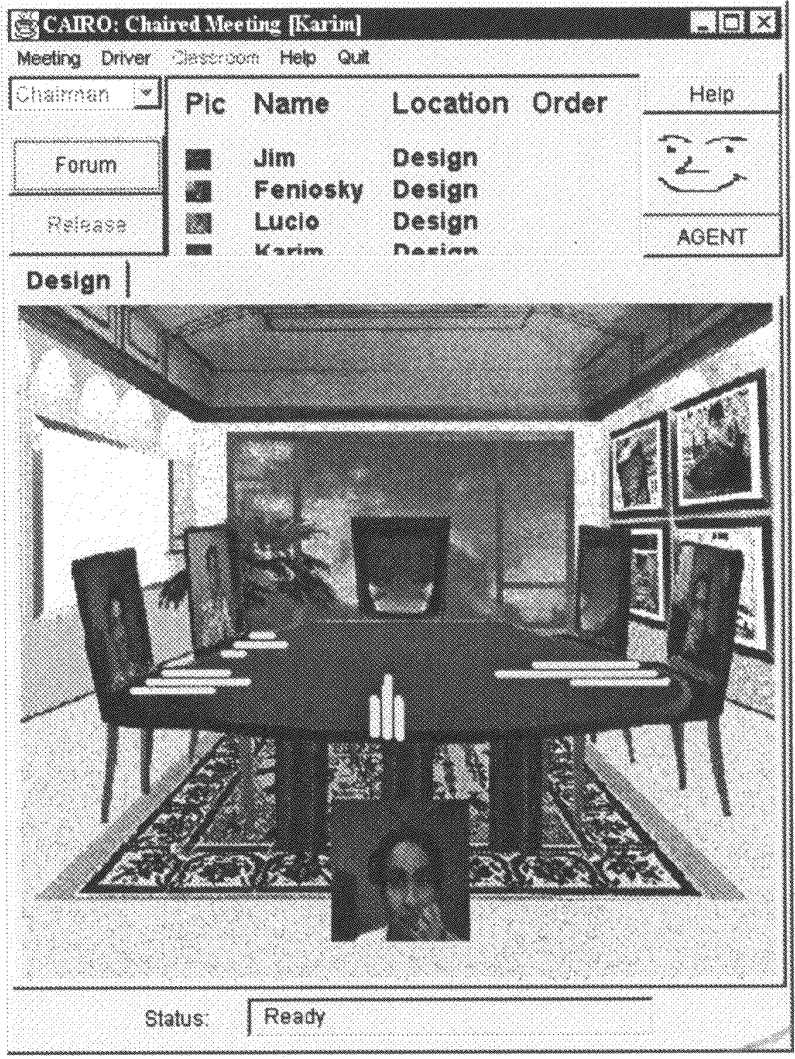

Fig. 9. Metaphor for representing degree of engagement.

rious issue if the floor transition is manual because social pressures will enforce honesty in proclaiming a degree of engagement. Finally, techniques need to be developed for detecting disfunctional group dynamics based on a thorough discourse analysis of various problem-solving situations.

\section{REFERENCES}

1. Ahuja, S. R. \& Ensor, J. R., Coordination and control of multimedia conferencing, IEEE Communications Magazine, 30 (5) (May 1992), 38-43.

2. Auramaki, E., Hirschheim, R. \& Lyytinen, K., Modelling offices through discourse analysis: A comparison and evaluation of SAMPO with OSSAD and ICN, Technical Report, University of Jyvaskyla, Helsinki, Finland, 1992.

3. Clark, H., Using Language, Cambridge University Press, Cambridge, England, 1996.

4. Cole, P. \& Nast-Cole, J., A primer on group dynamics for groupware developers, in Groupware: Software for ComputerSupported Collaborative Work, eds. D. Marca \& G. Bock, IEEE Computer Society Press, New York, 1992, pp. 44-57.

5. Craighill, E., Lang, R., Fong, M. \& Skinner, K., CECED: A system for informal multimedia collaboration, Technical Report, SRI International, Menlo Park, Calif., 1993.

6. Doyle, M. \& Straus, D., How to Make Meetings Work, Berkeley Books, Berkeley, Calif.,1993.

7. Duncan, S., On the structure of speaker-auditor interaction during speaking turns, Language in Society, 3 (1974).

8. Edelsky, C., Who's got the floor?, Language in Society, 10 (3), 383-421.

9. Egido, C., Video conferencing as a technology to support group work: A review of its failures, in Proceedings of the Second Conference on Computer-Supported Cooperative Work, ACM, Portland, Oregon, Sept 1988, pp. 13-24.

10. Ellis, C., Gibbs, S. \& Rein, G., Groupware: Some issues and experiences, Communications of the ACM, 34 (1) (1991), 3858.

11. Fisher, R. \& Ury, W., Getting to Yes, Penguin Books, New York, 1991.

12. Fitzpatrick, G., Kaplan, S. \& Mansfield, T., Physical spaces, virtual places and social worlds: A study of work in the virtual, in Conference on Computer-Supported Cooperative Work (CSCW '96), November 1996, ACM, Boston, Mass., pp. 334-43.

13. Fitzpatrick, G., Tolone, W. \& Kaplan, S., Work, locales and distributed social worlds, in Fourth European Conference on
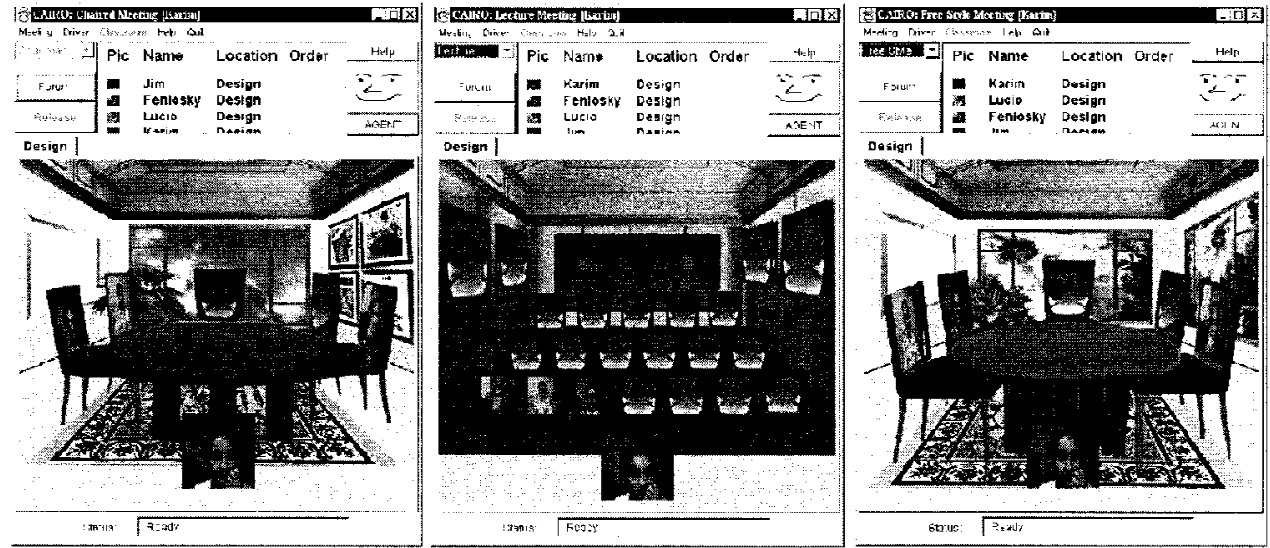

Fig. 10. Representing multiple floor control strategies: (a) chaired, (b) lecture, and (c) brainstorming. 
Computer-Supported Cooperative Work, Kluwer Academic Publishers, Boston, 1995, pp. 1-16.

14. Goodwin, C., Conversational Organization: Interaction between Speakers and Hearers, Academic Press, New York, 1981.

15. Harrison, S. \& Dourish, P., Re-placing space: The roles of place and space in collaborative systems, in Conference on ComputerSupported Cooperative Work (CSCW '96), November 1996, ACM, Boston, Mass., pp. 67-76.

16. Hiltz, S. R. \& Turoff, M., The Network Nation: Human Communication via Computer, MIT Press, Cambridge, Mass., 1993.

17. Hussein, K., Communication facilitators for a distributed collaborative engineering environment, Master's thesis, Massachusetts Institute of Technology, Cambridge, Mass., 1995.

18. Intel Corp., User's Guide: Intel Proshare Conferencing Products, 1996.

19. Ishii, H., Kobayashi, M. \& Arita, K., Interactive design of seamless collaboration media, Communications of the ACM, 37 (8) (1994), 84-92.

20. Kennedy, J. F., Contrasts in construction: A comparison between the construction industry, environment, and corporate culture in Japan and the United States, Technical Report, Intelligent Engineering Systems Laboratory, Massachusetts Institute of Technology, Cambridge, Mass., September 1996.

21. Levasseur, R. E., Breakthrough Business Meetings, Bob Adams, Inc., 1994.

22. McDaniel, S., Olson, G. \& McGee, J., Identifying and analyzing threads in computer-mediated and face-to-face conversations, in Conference on Computer-Supported Cooperative Work (CSCW '96), November 1996, ACM, Boston, Mass., pp. 39-47.

23. McNeill, D., Hand and Mind: What Gestures Reveal about Thought, University of Chicago Press, Chicago, Ill., 1992.

24. Microsoft Corp., Netmeeting home, http://www.microsoft.com/ netmeeting, 1997.

25. Murnighan, J. \& Conlon, D., The dynamics of intense work groups, Administrative Science Quarterly, 36 (2) (1991), 16586.

26. Nakanishi, H., Yoshida, C., Nishimura, T. \& Ishida, T., Freewalk: Supporting casual meetings in a network, in Conference on Computer-Supported Cooperative Work (CSCW'96), November 1996, ACM, Boston, Mass., pp. 308-14.

27. Netscape Corp., Welcome to Netscape Navigator Gold, Release 3.0., http://home.netscape.com/eng/mozilla/3.0, 1997.

28. Nunamaker, F. F., Dennis, A. R., Valacich, J., Vogel, D. R. \& George, J. F., Electronic meeting systems to support group work, Communications of the ACM, 34 (7) (1991), 40-61.

29. Olson, J., Olson, G. \& Meader, D., What mix of video and audio is useful for remote real-time work? in Computer Human Interaction (CHI '95), 1995, ACM, Denver, Colo., pp. 362-8.

30. Olson, J., Olson, G., Storrsten, M. \& Carter, M., Groupwork close up: A comparison of the group design process with and without a simple group editor, ACM Transactions on Information Systems, 11 (1994), 321-48.

31. Patton, B. R., Giffin, K. \& Patton, E. N., Communications within the group, in Decision-Making Group Interaction, Harper \& Row, New York, 1987.

32. Peña-Mora, F., Hussein, K. \& Sriram, R. D., CAIRO: A system for facilitating communication in a distributed collaborative engineering environment, Journal of Computers in Industry, 29 (1996), 37-50 (special collaborative engineering issue).

33. PictureTel Corp., PictureTel Products, http://www.picturetel. com/products.htm, 1997.

34. Roseman, M. \& Greenberg, S., Teamrooms: Network places for collaboration, in Conference on Computer-Supported Cooperative Work (CSCW '96), November 1996, ACM, Boston, Mass., pp. 325-33.

35. Sacks, H., Lectures on Conversation: Vols. I and II, Blackwell, Boston, 1995.

36. Schein, E. H., Process Consultation, Vol. II, Addison-Wesley Publishing Company, Reading, Mass., 1987.

37. Schein, E. H., Process Consultation, Vol. I, 2d Ed., AddisonWesley Publishing Company, Reading, Mass., 1988.

38. Silicon Graphics Corp., Inperson 2.2 Product Guide, http://www.sgi.com/Products/software/InPerson, 1997.

39. Srinivas, K., Reddy, R., Babadi, A., Kamana, S., Kumar, V. \& Dai, Z., MONET: A multimedia system for conferencing and application sharing in distributed systems, Technical Report CERC-TR-RN-91-009, West Virginia University, Concurrent Engineering Research Center, West Virginia University, Feb. 1992.

40. Stefik, M., Foster, G., Bobrow, D., Kahn, K., Lanning, S. \& Suchman, L., Beyond the chalkboard: Computer support for collaboration and problem solving in meetings, Transactions of the ACM, 30 (1) (1987), pp. 32-47.

41. Susskind, L. \& Cruikshank, J., Breaking the Impasse, Basic Books, New York, 1987.

42. Thorisson, K. R., Computational characteristics of multimodal dialogue, in AAAI Fall Symposium on Embodied Language and Action, Massachusetts Institute of Technology, Cambridge, Mass., November 1995.

43. Thorisson, K. R., Communicative humanoids: A computational model of psychosocial dialogue skills, unpublished MIT Media Lab dissertation, Massachusetts Institute of Technology, Cambridge, Mass., 1996.

44. Walton, R. \& Hackman, J., Designing Effective Work Groups, Jossey-Bass, San Francisco, 1986.

45. Yerian, K., Interactional synchrony in speech and gesture across crossed conversations, in Proceedings of the 34th Annual Meeting of the Association of Computational Linguistics, June 1996, ACL, Santa Cruz, Calif. 